\title{
The effect of age on fever response among nursing home residents with SARS-COV-2 infection
}

\author{
Moniyka Sachar ${ }^{1,2,3}$ (1) $\cdot$ Tom Bayer $^{2,3} \cdot$ Frank DeVone $^{2,3} \cdot$ Chris Halladay $^{2,3} \cdot$ Kevin McConeghy $^{2,3}$. \\ Salaheldin Elhamamsy ${ }^{2,3} \cdot$ Ashna Rajan ${ }^{2,3} \cdot$ Marilyne Cadieux ${ }^{2,3} \cdot$ Mriganka Singh $^{4} \cdot$ Aman Nanda $^{2,3}$. \\ James L. Rudolph ${ }^{2,3} \cdot$ Lynn McNicoll ${ }^{2,3} \cdot$ Sevdenur Cizginer ${ }^{2,3} \cdot$ Stefan Gravenstein ${ }^{2,3}$
}

Received: 26 August 2021 / Accepted: 5 December 2021 / Published online: 13 January 2022

(c) The Author(s), under exclusive licence to Springer Nature Switzerland AG 2022

\begin{abstract}
Over 15,000 veterans in 135 VA nursing homes were systematically tested for SARS-CoV-2 and had daily temperatures assessed from March to August, 2020. Lower baseline temperatures, and in SARS-CoV-2+, lower maximum temperatures were observed with advancing age. Clinicians should be aware of the potential diminished fever response in the elderly with SARS-CoV-2.
\end{abstract}

Keywords SARS-COV-2 $\cdot$ Covid $\cdot$ Aging

\section{Introduction}

Severe acute respiratory syndrome coronavirus 2 (SARS$\mathrm{CoV}-2$ ) infection manifests as either asymptomatic infection to any combination of systemic, upper respiratory, or gastrointestinal symptoms. Given this wide range, fever is a commonly monitored indicator of active SARS-CoV-2 infection in nursing homes and other high-risk settings. It has been postulated that the aging population has a lower baseline temperature ( $\mathrm{Tbl}$ ), defined as resting temperature in a non-infected or noninflammatory state. This trend has been attributed to diminished thermoregulatory responses, decreased production and transport of pyrogens, and less thermogenic brown fat [1-3]. In 2020, the CDC recommended a single temperature above $37.8^{\circ} \mathrm{C}$ or $1.1^{\circ} \mathrm{C}$ above baseline, or multiple readings above $37.2^{\circ} \mathrm{C}$, be considered a sign of infection in the elderly [4]. We investigated whether

Moniyka Sachar

sacharmoniyka@gmail.com

1 New York University Langone Medical Center, New York, USA

2 Providence VA Medical Center COIN-LTSS, Providence, USA

3 Alpert Medical School of Brown University, Providence, USA

4 University Hospitals-Case Western Reserve University, Cleveland, USA increasing age contributes to a lower maximum temperature (Tmax) in SARS-COV-2, and thus a blunted fever response during infection. We characterized the relationship between age and body temperature fluctuations among the SARSCOV-2 infected and uninfected elderly population.

\section{Methods}

This study was approved by the Providence Veterans Administration (VA) Medical Center's Institutional Review Board. We retrospectively evaluated temperatures from residents in Veterans Administration's (VA) community living centers (CLC) during the period of March 1, 2020 to August 5, 2020. During this period, regardless of symptomatology, residents were systematically tested for SARS-COV-2 infection and had temperatures recorded at least daily in the electronic medical records (EMR) system.

We included residents who were 50 years and above, tested for SARS-CoV-2 infection via reverse transcription polymerase chain reaction (RT-PCR) testing, and had daily or more temperature readings. We defined a testing period as three days before and after a SARS test, Tmax as the maximum temperature in this period. We defined Tbl as the mean temperature of the last 5 temperatures 2 weeks prior to testing period and Tdev as the maximum deviation from Tbl in the testing period. 
Using ICD-10 codes, we retrospectively identified common comorbidities among residents listed as a diagnosis a year prior to the study period, in March of 2019. We screened the EMR for anti-pyretic use, specifically acetaminophen and NSAIDS, including all alternative names, administered at least once at the minimum or recommended dosage during the study period.

We categorized residents into three age categories, specifically ages 50-69, 70-89, and 90+, and further stratified residents based on whether they were SARS + or SARS- . We used chi-square analysis to compare the Tmax, Tdaily, Tbl, and Tdev across these three age groups and by SARSCov-2 status. We further described antipyretic use and the diagnosis of major co-morbidities, specifically diabetes mellitus (type 1 or type 2), hypertension, and pulmonary disease (COPD or asthma), across these three age categories. We used standard deviation, $\mathrm{p}$ value (set at 0.05 ) and confidence intervals to compare these groups. Continuous variables were compared with ANOVA tests and categorical variables compared with chi-square tests.

\section{Results}

A total of 15,043 residents underwent SARS-CoV-2 testing between March and December of 2020 at a VA CLC. Of these, 13,410 had daily or more temperature readings, and 13,042 residents were at or above the age of 50. These 13,042 residents formed six groups based on the age category (50-69, 70-89, and 90+) and SARS-CoV-2 infection status (positive or negative). A total of 1767 (16\%) residents had a positive SARS-CoV-2 test.

Across the three age categories, there was no difference in the proportion of antipyretic use which could skew temperature readings (Table 1). The proportion of residents diagnosed with diabetes mellitus, hypertension, or pulmonary disease was the highest in the 70-89 category and the lowest in the $90+$ category for each condition, respectively. The mean BMI decreased with each increasing age category. Despite having a lower incidence of chronic disease, the $90+$ category had the greatest proportions of SARS positivity, death to any cause, death due to SARS-Cov-2 infection.
Table 1 Demographics by Age Category and Temperature Trends by SARS Status

\begin{tabular}{lllll}
\hline Predictor & Ages 50-69 & Ages 70-89 & Ages 90+ & $P$ value \\
\hline Demographics & & & & \\
$N$ & 4,014 & 7,932 & 1,096 & \\
Death (any cause) & $563(14.03 \%)^{\mathrm{a}}$ & $2,013(25.88 \%)$ & $514(46.90 \%)$ & $<0.001$ \\
Death (SARS-Cov-2) & $62(1.54 \%)$ & $336(4.24 \%)$ & $99(9.03 \%)$ & $<0.001$ \\
Mean BMI & $29.02(8.06)^{\mathrm{b}}$ & $28.16(7.01)$ & $25.23(5.05)$ & $<0.001$ \\
Anti-pyretic use & $2,975(74.12 \%)$ & $5,794(73.05 \%)$ & $797(72.72 \%)$ & 0.406 \\
Diabetes Mellitus & $1,649(41.08 \%)$ & $3,468(43.73 \%)$ & $287(26.19 \%)$ & $<0.001$ \\
Hypertension & $2,911(72.52 \%)$ & $6,061(76.42 \%)$ & $772(70.44 \%)$ & $<0.001$ \\
Pulmonary Disease & $1,491(37.14 \%)$ & $3,296(41.56 \%)$ & $374(34.12 \%)$ & $<0.001$ \\
SARS-CoV-2 positive & & & & \\
$N$ & $439(10.94 \%)$ & $1,137(14.33 \%)$ & $191(17.43 \%)$ & \\
Tbl & $36.59(0.24)$ & $36.55(0.24)$ & $36.55(0.24)$ & 0.004 \\
$T$ max & $37.74(0.83)$ & $37.76(0.77)$ & $37.69(0.75)$ & 0.003 \\
$T$ dev & $1.15(0.85)$ & $1.21(0.79)$ & $1.15(0.75)$ & 0.132 \\
SARS-CoV-2 Negative & & & & \\
$N$ & $3575(89.06 \%)$ & $6795(85.66 \%)$ & $905(82.57 \%)$ & \\
Tbl & $36.59(0.24)$ & $36.56(0.24)$ & $36.52(0.24)$ & 0.004 \\
$T$ max & $37.08(0.49)$ & $37.09(0.49)$ & $37.07(0.47)$ & 0.253 \\
$T$ dev & $0.50(0.50)$ & $0.53(0.50)$ & $0.55(0.48)$ & 0.850 \\
\hline
\end{tabular}

$N$ sample size; $B M I$ body mass index, $T b l$ baseline temperature as a mean calculated over five days, 2 weeks prior to the testing period. Testing period defined as three days before and after SARS-Cov-2 test, Tmax maximum temperature recorded during the testing period, Tdev maximum deviation from baseline temperature during the testing period

Antipyretic use included acetaminophen, ibuprofen, aspirin, and other NSAIDs. Diagnoses of diabetes mellitus, hypertension, and pulmonary disease were based on the diagnoses recorded at least 1 year prior to study enrollment

${ }^{\text {a }}$ Proportions reported as number (percentage)

${ }^{\mathrm{b}}$ Means reported as mean (standard deviation) 
Table 1 also depicts the trends in Tmax and Tdaily by age category and by SARS status by "all" tests in the testing period. The mean Tbl declined across increasing age categories for both SARS $+(P=0.004)$ and SARS $-(P=0.004)$ residents (Table 1). In SARS + residents the mean Tmax declined across increasing age categories, with $37.74{ }^{\circ} \mathrm{C}$ at age $50-69,37.76{ }^{\circ} \mathrm{C}$ at age $70-89$, and $37.69{ }^{\circ} \mathrm{C}$ at age $90+(P=0.003)$, with all Tmax temperatures below the traditional cutoff of $38{ }^{\circ} \mathrm{C}$. No similar trend in Tmax was observed in SARS - residents $(P=0.253)$. For both SARS + and SARS - residents, there was no difference in the Tdev across age categories.

\section{Discussion/conclusion}

Lower Tbls, and with SARS+, lower Tmax were observed with advancing age in $\mathrm{NH}$ residents. This trend in lower baseline temperatures with age may be attributed to diminished thermoregulatory responses and less thermogenic brown fat seen as part of the normal aging process [1-3]. The trend in lower maximum temperatures during SARSCov-2 infection may be due to decreased production and transport of pyrogens with aging, as has been speculated to explain diminished fever responses in other infections [1-3]. In relation to $2020 \mathrm{CDC}$ guidance, the Tmax across all age categories was less than the recommended one time $37.8^{\circ} \mathrm{C}$ cutoff, but the deviation from baseline temperatures matched the recommended $1.1{ }^{\circ} \mathrm{C}$. Given Tdev remained consistent across age categories, our data supports using deviation from baseline temperature (i.e., $1.1^{\circ} \mathrm{C}$ above baseline) as a potential metric when identifying temperatures concerning for infection in the elderly population.

Survivorship bias, as is commonly seen in studies of aging, may explain the trend of lower BMI and lower incidence of diabetes mellitus, hypertension, and pulmonary disease in older patients. Patients who live to 90 years and beyond may be less likely to have metabolic dysfunction and chronic disease, hence their longevity. This trend may also explain our findings of "older is colder." For example, those who survive beyond the age of 90 may have lower brown fat secondary to lower BMIs, and lower pyrogen production secondary to lower incidence of chronic inflammatory conditions. Another explanation for colder body temperatures is the reported slower metabolism and sedentary lifestyle seen with aging [1-3]. We should acknowledge our population of nursing home residents may be more likely to be sedentary than an age-matched nonresidents, thus contributing to lower basal metabolic rates and temperatures overall.

Clinically, our data suggest the utility of relative body temperature, based on the temperature variability of each individual at baseline, for greater precision when suspecting outlier, age-adjusted temperatures in SARS-CoV-2 or other infections. Future projects may consider the potential for electronic clinical records that can track and flag outlier age-adjusted temperatures and temperature deviations, and clinicians should continue to consider the importance of age and individual metabolic variations when evaluating a patient for "fever".

Funding The study was supported by Veterans Administration (VA) Health Services Research and Development Center of Innovation in Long Term Services and Supports (CIN 13-419 and C19 20-213) and the National Institute of Aging (3P01AG027296-11S2) and approved by the Providence Veterans Administration Medical Center (VAMC) Institutional Review Board.

\section{Declarations}

Conflict of interest All authors declare they have no conflicts of interest with publication of this paper.

Human and animal rights All procedures performed in studies involving human participants were in accordance with the ethical standards of the institutional and/or national research committee and with the 1964 Helsinki declaration and its later amendments or comparable ethical standards.

Informed consent Informed consent was obtained from all individual participants included in the study. This study was approved by the Providence Veterans Administration Medical Center (VAMC) Institutional Review Board.

\section{References}

1. Lu S-H, Leasure A, Dai Y-T (2009) A systematic review of body temperature variations in older people. J Clin Nurs 19:4-16. https://doi.org/10.1111/j.1365-2702.2009.02945.x

2. Norman DC (2000) Fever in the elderly. Clin Infect Dis 31:148151. https://doi.org/10.1086/313896

3. El Chakhtoura NG, Bonomo RA, Jump RLP (2017) Influence of aging and environment on presentation of infection in older adults. Infect Dis Clin N Am 31:593-608. https://doi.org/10.1016/j.idc. 2017.07.017

4. CDC. COVID-19 and Your Health (2020) Centers for Disease Control and Prevention. Published February 11, 2020. https:// www.cdc.gov/coronavirus/2019-ncov/need-extra-precautions/ older-adults.html. Accessed 29 Dec 2020

Publisher's Note Springer Nature remains neutral with regard to jurisdictional claims in published maps and institutional affiliations. 